\title{
Caracterización genética molecular de materiales procesados de Stevia rebaudiana utilizando la técnica de microsatélites
}

\author{
Molecular-genetic characterization of Stevia rebaudiana \\ processed materials using microsatellite technique
}

Giovanni Garro-Monge'

Karol Jiménez-Quesada²

Silvana Alvarenga-Venutolo ${ }^{3}$

Fecha de recepción: 21 de enero del 2014

Fecha de aprobación: 08 de abril del 2014

Garro-Monge, G; Jiménez-Quesada. K; Alvarenga-

Venutolo, S. Caracterización genética molecular de materiales procesados de Stevia rebaudiana utilizando la técnica de microsatélites Tecnología en Marcha. Vol. 27, N 3, Julio-Setiembre 20 I4. Pág 32-40.

I Centro de investigación en Biotecnología (CIB), Instituto Tecnológico de Cota Rica. Costa Rica. Correo electrónico: ggarro@itcrac.cr.

2 Centro de investigación en Biotecnología (CIB), Instituto Tecnológico de Cota Rica. Costa Rica. Correo electrónico: kjimenez@itcr.ac.cr.

3 Centro de investigación en Biotecnología (CIB), Instituto Tecnológico de Cota Rica. Costa Rica. Correo electrónico: salvarenga@itcr.ac.cr. 


\section{Palabras clave}

Caracterización genética; cuantificación de ácidos nucleicos; ISSR; marcadores moleculares; microsatélites, NanoDrop; PCR; Stevia rebaudiana.

\section{Resumen}

Stevia rebaudiana es una planta herbácea perenne que pertenece a la familia Asteraceae. Es originaria del norte de Paraguay y crece en climas tropicales y subtropicales. Debido a la dulzura natural de sus hojas, se le conoce como edulcorante natural, sin calorías y con muchas aplicaciones en la industria alimentaria. Por lo tanto, resulta de gran importancia comercial contar con técnicas que permitan determinar la calidad y pureza de la materia prima procesada en polvo para la producción de edulcorantes. Se ha recurrido a la implementación de técnicas moleculares mediante marcadores ISSR, que permiten amplificar las secuencias que se encuentran entre los microsatélites, con lo que se obtiene un patrón de bandas característico de cada organismo.

En este estudio se analizó la amplificación de los ISSR S3, S4, S5, SIO, SII, SI 2 y SI 5 a partir de muestras de ADN de S. rebaudiana, provenientes de plantas cultivadas en invernadero. Las muestras de ADN se obtuvieron mediante tres diferentes protocolos de extracción a partir de material seco y fresco de la planta. De acuerdo con los resultados obtenidos, los ISSR S3, S4, S5 y SI I representan una buena opción para la detección de polimorfismos en esta especie, y por tanto para la identificación molecular de la especie. Se debe comprobar si los ISSR SIO, SI2 y SI5 son capaces de amplificar segmentos de ADN de Stevia, ya que bajo las condiciones de trabajo de esta investigación no se logró la amplificación de ADN con estos imprimadores. Se considera que los ISSR son marcadores universales y se podría identificar individualmente cada cultivar de Stevia que vaya a destinarse a la producción comercial de esteviósidos, pero es necesario repetir los protocolos descritos con diferentes variedades e incluso se puede trabajar con diferentes especies del género Stevia, para obtener una caracterización general.

\section{Key words}

Genetic characterization; nucleic acid quantification; ISSR; molecular markers; microsatellites; NanoDrop; PCR; Stevia rebaudiana.

\begin{abstract}
Stevia rebaudiana is a herbaceous and perennial plant belonging to the Asteraceae family, it is native of Paraguay and grows in tropical and subtropical climates. Due to the natural sweetness of its leaves, is known as a natural sweetener with no calories and with many applications in food industry. Therefore, it is of great commercial importance to have techniques for determinate the quality and purity of the raw material processed into powder for the production of sweeteners. Been used for the implementation of molecular markers ISSR, enabling amplify sequences that are between the microsatellites, which yields a characteristic banding pattern for each organism.
\end{abstract}

In this study we examined the amplification of ISSR S3, S4, S5, SI O, SII, SI 2 and SI 5 from DNA samples of $S$. rebaudiana, from greenhouse-grown plants. DNA samples were obtained by three different extraction protocols from dry and fresh material from the plant. According to the results, the ISSR S3, S4, S5 and SII are a good choice to detect polymorphisms in this species, and therefore the molecular identification of the species. It is necessary to check whether the ISSR SIO, SI2 and SI5 are able to amplify DNA segments in stevia, since under the conditions of this research work was not successful DNA amplification with these primers. It is considered that ISSR markers are universal and could be used to identify every stevia cultivate for commercial production of stevioside, but it is necessary to repeat the protocols described with different varieties and work with different species of the genus Stevia sp., to obtain a general characterization . 


\section{Introducción}

La especie Stevia rebaudiana, perteneciente a la familia Asteraceae y originaria del norte de Paraguay y Suramérica, es una planta herbácea, perenne, que crece en climas tropicales y subtropicales (Shreeder et al., 2008). Debido a la dulzura natural de sus hojas, S. rebaudiana ha llamado la atención en los campos científicos e industriales como un edulcorante natural sin calorías con muchas aplicaciones en la industria alimentaria. Las hojas contienen glucósidos, que comprenden el esteviósido, rebaudiosidos $A$, B, C, D, E y F y dulcósido A (Karaköse, Rakesh y Kuhnert, 20II).

Hasta la fecha se han identificado más de 100 compuestos en S. rebaudiana y los esteviol glucósidos varían ampliamente su concentración, dependiendo del genotipo y el medio ambiente de producción de la planta. Actualmente esta planta se cultiva comercialmente en varios países, especialmente Japón, Taiwán, Corea, Tailandia e Indonesia (Prakash, Upreti y Prakash, 201 I).

La especie ha cobrado gran importancia comercial en los últimos años, por considerarse que sus extractos son un exitoso sustituto de la sacarosa, ante lo cual es necesario contar con herramientas que permitan determinar la identidad genética del material que se utilizará en la producción de edulcorantes, de tal forma que corresponda a la especie y/o variedad necesaria, como Stevia rebaudiana var. Bertoni. Debido al procesado en materia molida y seca, su contaminación con otros materiales podría ser frecuente, así como la probabilidad de ser confundida durante los envíos entre los centros de la cadena productiva. Por otro lado, cabe mencionar que existen otras variedades, como Stevia rebaudiana var. morita, o incluso con otras especies como Stevia eupatoria, Stevia ovata y Stevia plummerae, que no presentan un valor comercial (Chávez y Chavarría 20I I) y que podrían ser usadas para suplantar a la variedad comercial de forma fraudulenta.

Con este objetivo y gracias al avance en la biología molecular, se han desarrollado métodos de identificación y caracterización basados en el uso de marcadores moleculares, los cuales se definen como cualquier diferencia no típica controlada genéticamente, es decir, moléculas de proteína, ARN o ADN, de tamaño o peso molecular conocido que sirven para monitorear o calibrar la separación de las mismas moléculas utilizando electroforesis o cromatografía, para diferenciar individuos; estos marcadores son neutrales a las interacciones entre la expresión genética y el ambiente, presentan mayor segregación o polimorfismo que los morfológico y pueden ser evaluados desde los primeros estados de desarrollo de los organismos (Azofeifa, 2006; Sánchez y Jiménez, 2009).

Entre estos marcadores están: los polimorfismos de la longitud de los fragmentos de restricción (RFLP), amplificación aleatoria del ADN polimórfico (RAPD), polimorfismo en la longitud de los fragmentos amplificados (AFLP), secuencias simples repetidas (SSR) o microsatélites y secuencias intercaladas entre los microsatélites o ISSR (por su nombre en inglés, Interspread single sequence repeats) (Sambrook y Russell 200 I).

Los marcadores ISSR fueron desarrollados por Zietkewicz y colaboradores en 1994 y la técnica se fundamente en la amplificación por PCR utilizando un solo primer de 14 pb o más, que es complementario a secuencias en los microsatélites; la amplificación se da si el imprimador se puede unir adecuadamente a dos microsatélites que tienen una distancia de separación entre sí de 100 a 2500 pb, lo que permite amplificar las secuencias que se encuentran entre los microsatélites, con lo que se obtiene una patrón de bandas característico de cada organismo (Zietkewicz, Rafalski y Labuda, 1994; Chia 2009; Aga, Bekele y Bryngelsson, 2005; Masumbuko y Bryngelsson 2006).

Para la caracterización se debe realizar una amplificación de secuencias de ADN por la reacción en cadena de la polimerasa (PCR) utilizando imprimadores anclados o no anclados, la separación de los productos mediante electroforesis en geles de agarosa, poliacrilamida o geles de secuenciación (Chia, 2009; Azofeifa, 2006), con lo cual se puede visualizar por la formación de un patrón de bandas, que se debe a la presencia o ausencia de elementos genómicos reconocidos por el imprimador y a la longitud de las secuencias intermedias amplificadas, a este patrón se le llama huella genética, característica para esta especie (Chia, 2009; Azofeifa, 2006; Zietkiewicz et al., 1994).

Es por estas razones que se pretende utilizar los marcadores ISSR para caracterizar material fresco y molido de S. rebaudiana proveniente de cultivo in vitro e invernadero del Centro de Investigación en Biotecnología (CIB) del Instituto Tecnológico de Costa Rica. 


\section{Materiales y métodos}

Material vegetal: Las plantas de Stevia rebaudiana var. Bertoni utilizadas para la extracción de ADN procedían de los invernaderos y cuartos de crecimiento controlados del CIB. Para las extracciones de ADN a partir de material fresco de $S$. rebaudiana se seleccionaron hojas de material cultivado in vitro en medio semisólido y sometido a una temperatura controlada de $25 \pm 2{ }^{\circ} \mathrm{C}$, así como de material procedente de invernadero. Por otra parte, para la obtención de material seco y molido, se procedió a recolectar 100 gramos de hojas frescas de esta especie provenientes de invernadero, para ser sometidas a un proceso de secado a una temperatura de $45^{\circ} \mathrm{C}$ durante 48 horas, para luego moler el tejido, obteniéndose un total de 20 gramos de peso seco.

Extracciones de ADN: El material vegetal, tanto fresco como seco y molido, fue utilizado para la extracción de ADN mediante los protocolos CTAB, Bench Protocol del DNeasy Plant MiniKit marca QIAGEN y Isolating Genomic DNA from Plant Tissue del Wizard Genomic DNA Purification Kit marca PROMEGA. El material seco se pesó hasta una cantidad de $50 \mathrm{mg}$ para ser utilizados directamente en las extracciones siguiendo los diferentes protocolos. Del material fresco se pesaron $40 \mathrm{mg}$ de hoja para ser utilizados en el protocolo QIAGEN, $100 \mathrm{mg}$ para el protocolo Wizard y $50 \mathrm{mg}$ para el protocolo CTAB. El material fresco requirió ser colocado inicialmente en un tubo Eppendorf para macerarlo con nitrógeno líquido en un macerador Retsch, mediante tres repeticiones de 25 golpes por segundo durante un minuto, hasta obtener un polvo fino de hoja. Una vez preparada la muestra, se procedió a continuar con los protocolos correspondientes.

Cuantificación de ADN: Todas las muestras de ADN fueron sometidas a una electroforesis en gel de agarosa al $1 \%$ teñido con gel red $10000 X$, mediante una corrida de 30 minutos a $100 \mathrm{~V}$, para visualizar la presencia/ausencia del ácido nucleico en transiluminador. En las ocasiones en que la banda de ADN fue visible en el gel, se procedió a cuantificar la muestra mediante el uso del equipo NanoDrop ${ }^{\circledR}$ del Centro de Investigación en Biología Celular y Molecular (CIBCM) de la Universidad de Costa Rica. Se seleccionaron las muestras con mayor concentración de ADN para utilizarse en los análisis mediante ISSR.

Análisis PCR-ISSR: Se utilizaron siete primers ISSR basados en repeticiones de dos, cuatro o cinco bases, los cuales fueron reportados y utilizados por Heikal et al. (2008) en S. rebaudiana; estos se presentan en el cuadro I. Las reacciones de PCR consistieron en la mezcla de 50 o 60 ng de ADN, IX de Buffer PCR (Fermentas), 2 mM de $\mathrm{MgCl}_{2}$ (Fermentas), $200 \mu \mathrm{M}$ de dNTPs (Fermentas), I,25 $\mu \mathrm{M}$ del marcador ISSR y IU de Taq polimerasa (Fermentas), hasta alcanzar los $25 \mu \mathrm{l}$ de reacción. El protocolo de termociclador se basa en el propuesto por Yao et al. (1999), que se inicia con una desnaturalización de las hebras a $94^{\circ} \mathrm{C}$ por 2 min, seguida de 40 ciclos de 10 s a 94 ${ }^{\circ} \mathrm{C}, 30$ s a $36{ }^{\circ} \mathrm{C}$ y 65 s a $72{ }^{\circ} \mathrm{C}$ y la extensión final de los productos se dio a $72{ }^{\circ} \mathrm{C}$ por 10 min para culminar con el almacenamiento a $4{ }^{\circ} \mathrm{C}$. Se realizó

Cuadro I. Código, nombre y secuencia de siete imprimadores tipo marcadores ISSR utilizados en la caracterización molecular de S. rebaudiana.

\begin{tabular}{|c|c|c|}
\hline Código & Nombre & Secuencia \\
\hline S3 & ISSR 844B & 5'-CTCTCT CTCTCT CTC TGC-3' \\
\hline S4 & ISSR 17898A & 5'- CAC ACA CAC ACA AC -3' \\
\hline S5 & ISSR 17898B & 5'- CAC ACA CAC ACA GT -3' \\
\hline SI0 & ISSR HB-10 & 5'- GAG AGA GAG AGA CC -3' \\
\hline SII & ISSR HB-11 & 5'- GTG TGT GTG TGT CC -3' \\
\hline SI2 & ISSR HB-12 CAC CAC CAC GC -3' \\
\hline SI5 & ISSR HB-15 & 5'- GTG GTG GTG GC -3' \\
\hline
\end{tabular}

Fuente: Heikal et al. 2008. 
una modificación al protocolo anterior, cambiando la temperatura de hibridación a $44{ }^{\circ} \mathrm{C}$, que era el promedio de las temperaturas específicas para cada imprimador en estudio. Los productos amplificados con cada ISSR fueron separados mediante electroforesis en gel de agarosa al I,5\% teñido con gel red I0000X, mediante una corrida a 100 v durante 30 min, para visualizar la presencia/ausencia en transiluminador de los productos amplificados.

\section{Resultados y discusión}

las muestras procedentes de las extracciones de ADN se visualizaron mediante electroforesis en gel de agarosa y se seleccionaron las ocho muestras que habían generado las bandas de mayor intensidad, como se muestra en el cuadro 2. Posteriormente se continuó con la cuantificación de dichas muestras con el equipo NanoDrop $®$ y los resultados se presentan en el cuadro 3.

Como es posible deducir del cuadro anterior, todos los protocolos empleados para la extracción de $A D N$ de $S$. rebaudiana dieron positivo en la presencia de bandas intensas en al menos una muestra $y$, por ende, fueron seleccionadas para su cuantificación. A través de los años las metodologías para la obtención de ácidos nucleicos se han ido optimizando. Las extracciones con fenol/cloroformo estuvieron entre las primeras metodologías utilizadas (Collette et al., 2006; Hiesh et al., 2005; Lin et al., 2005), pero actualmente en el mercado se encuentra una gran cantidad de kits que permiten la obtención de ácidos nucleicos en poco tiempo mediante protocolos sencillos. La mayoría de ellos se basa en la utilización de columnas de silica en las cuales, en presencia de altas concentraciones de sales caotrópicas, se adhiere el ADN, que luego es recuperado por arrastre (Ivanova et al., 2006).

Debido a lo anterior se quiso comprobar la eficacia de los tres protocolos propuestos, de manera que se lograra obtener ADN de alta calidad para realizar las pruebas de caracterización mediante el uso de los marcadores ISSR, para evitar sesgos en los resultados por interferencias o contaminaciones de los ácidos nucleicos.

Según los datos presentados anteriormente, utilizando el Genomic DNA Purification Kit (WIZARD) se obtuvo la mayor concentración de $A D N$, seguido por el método CTAB y finalmente el Kit DNeasy Plant Mini (QIAGEN), el cual presentó el ADN en menor concentración.

No obstante, en cuanto a la calidad del ADN, se tiene que cinco de las ocho muestras presentaron una pureza aceptable del ADN extraído, ya que la relación 260/280 se encuentra en el rango de 1,6 a 1,9. Las muestras WIZARD 3, y WIZARDINVER2 estuvieron por debajo del rango, mientras que la muestra QIAGENINVITRO2 superó el rango aceptable. La razón por la cual la absorción de luz

Cuadro 2. Muestras de ADN de S. rebaudiana procedentes de tres protocolos de extracción distintos, seleccionadas para su cuantificación en el equipo NanoDrop®.

\begin{tabular}{|c|c|c|c|}
\hline $\begin{array}{c}\text { Presentación del material } \\
\text { vegetal }\end{array}$ & Protocolo de extracción & $N^{\circ}$ de muestra & Identificación de la muestra \\
\hline \multirow{3}{*}{ Molido } & CTAB & Muestra I & CTABI \\
\hline & Bench Protocol (QIAGEN) & Muestra 2 & QUIAGEN2 \\
\hline & Wizard (PROMEGA) & Muestra 3 & WIZARD3 \\
\hline \multirow{3}{*}{ Fresco } & CTAB & Muestra 2 invernadero & CTABINVER2 \\
\hline & Bench Protocol (QIAGEN) & $\begin{array}{c}\text { Muestra } 2 \text { in vitro } \\
\text { Muestra } 2 \text { invernadero }\end{array}$ & $\begin{array}{l}\text { QIAGENINVITRO2 } \\
\text { QIAGENINVER2 }\end{array}$ \\
\hline & Wizard (PROMEGA) & $\begin{array}{c}\text { Muestra } 2 \text { in vitro } \\
\text { Muestra } 2 \text { invernadero }\end{array}$ & $\begin{array}{l}\text { WIZARDINVITRO2 } \\
\text { WIZARDINVER2 }\end{array}$ \\
\hline
\end{tabular}


Cuadro 3. Resumen de los resultados de la cuantificación de ocho muestras de ADN de S. rebaudiana procedentes de tres protocolos de extracción distintos.

\begin{tabular}{|c|c|c|c|c|c|c|}
\hline & $\begin{array}{l}\text { Identificación de la } \\
\text { muestra }\end{array}$ & $\begin{array}{c}\text { Concentración de } \\
\text { ADN }(n g / \mu l)\end{array}$ & $\begin{array}{l}\text { Absorbancia a } \\
260 \mathrm{~nm}\end{array}$ & $\begin{array}{l}\text { Absorbancia a } \\
280 \mathrm{~nm}\end{array}$ & $\begin{array}{l}\text { Relación } \\
260 / 280\end{array}$ & Factor \\
\hline \multirow[t]{8}{*}{ AGUA } & CTABI & 191,7 & 3,835 & 2,059 & 1,86 & 50 \\
\hline & QIAGEN2 & 8,3 & 0,166 & 0,102 & 1,62 & 50 \\
\hline & WIZARD3 & 77,3 & 1,546 & 1 & ।,55 & 50 \\
\hline & CTABINVER2 & 57,2 & I, I 44 & 0,612 & ।,87 & 50 \\
\hline & QIAGENINV2 & 33,8 & 0,677 & 0,363 & 1,86 & 50 \\
\hline & QIAGENINVITRO2 & 6,3 & 0,126 & 0,053 & 2,38 & 50 \\
\hline & WIZARDINVITRO2 & 101 & 2,021 & 1,122 & 1,8 & 50 \\
\hline & WIZARDINVER2 & 864,4 & 17,29 & 13,32 & 1,3 & 50 \\
\hline \multirow[t]{8}{*}{$\mathrm{TE}(\mathrm{IX})$} & CTABI & 191,1 & 3,823 & 2,046 & 1,87 & 50 \\
\hline & QIAGEN2 & 6,5 & 0,129 & 0,074 & 1,75 & 50 \\
\hline & WIZARD3 & 75 & ।,499 & 0,96 & 1,56 & 50 \\
\hline & CTABINVER2 & 56,7 & 1,134 & 0,606 & ।,87 & 50 \\
\hline & QIAGENINVER2 & 33,7 & 0,675 & 0,373 & $|, 8|$ & 50 \\
\hline & QIAGENINVITRO2 & 6,2 & 0,125 & 0,055 & 2,27 & 50 \\
\hline & WIZARDINVITRO2 & 99,4 & 1,987 & ।,108 & 1,79 & 50 \\
\hline & WIZARDINVER2 & 849,5 & 16,99 & 13,19 & 1,29 & 50 \\
\hline
\end{tabular}

UV es uno de los métodos más utilizados para la cuantificación del ADN es que los nucleótidos poseen máximos de absorción de alrededor de $260 \mathrm{~nm}$, por lo que es posible obtener una estimación precisa de la concentración de una muestra. Sin embargo, para evaluar la pureza de la muestra debe determinarse la proporción OD 260nm/OD $280 \mathrm{~nm}$, para contrarrestar la posibilidad de que la lectura a $260 \mathrm{~nm}$ se altere por la presencia de contaminación por proteínas o solventes orgánicos que absorben a longitudes de onda cercanas (López, 2007; Wilfinger et al., 1997).

Para efectos de esta investigación, para la caracterización molecular de $S$. reabaudiana se seleccionaron las muestras CTABI, WIZARD3, CTABINVER2, WIZARDINVITRO2 y WIZARDINVER2 por ser las que presentaron mayor concentración de ADN. Las muestras se trabajaron en las reacciones PCR/ISSR en dos concentraciones, 50 y $60 \mathrm{ng}$.
Con el marcador S3, se logró una amplificación del ADN utilizando las dos concentraciones de ADN para el material fresco proveniente de cultivo in vitro, no obstante, al emplear 60 ng de ADN en la reacción, se logró además una amplificación de la muestra CTABINVER2; lo anterior se muestra en la figura 1.

Con el marcador S4, se logró una amplificación del ADN utilizando $60 \mathrm{ng}$ de ADN de la muestra CTABINVER2 únicamente, como se observa en la figura 2.

Una vez comprobada la aparición de bandas claramente visibles, con el protocolo de termociclado propuesto, para los productos de PCR empleando los imprimadores ISSR S3 y S4, se continuó con la ejecución de reacciones utilizando los imprimadores S5, SI0, SI I, SI 2 y SI 5 por separado. En la figura 3 se muestran los resultados de la amplificación con los siete imprimadores en estudio. 


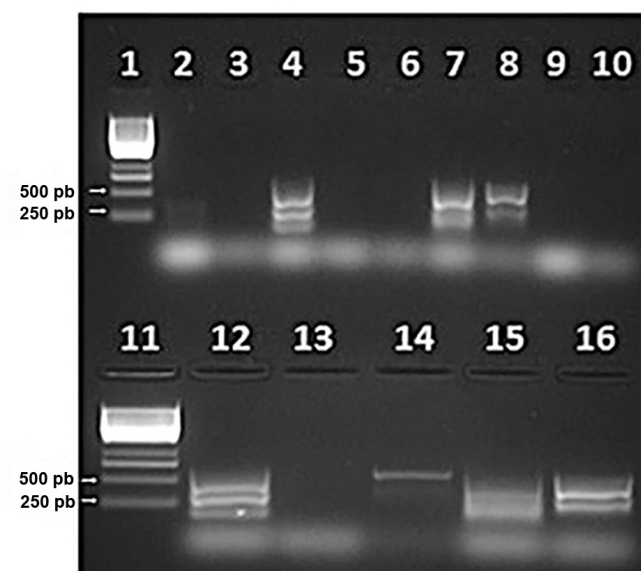

Figura I. Producto de PCR amplificado con el ISSR S3: I y | |) Marcador de peso molecular Gene Ruler, 2 y 9) ADN CTABI (50 y 60 ng), 3 y 10) ADN WIZARD3 (50 y 60 ng), 4 y I2) ADN WIZARDINVITRO2 (50 y 60 ng), 5 y 13) ADN WIZARDINVER2 (50 y 60 ng), 6 y 14) ADN CTABINVER2 (50 y 60 ng), 7 y 15 ) ADN Sechium edule, 8 y 16) ADN Uncaria tomentosa.

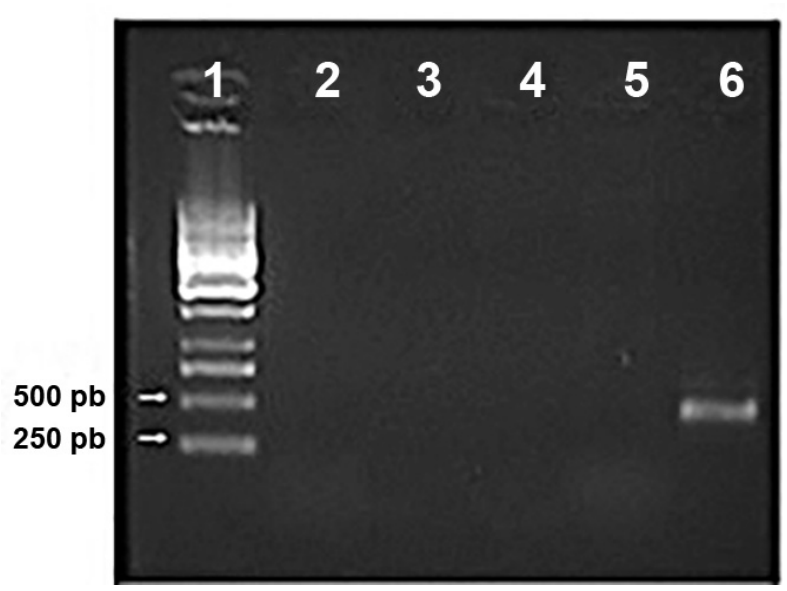

Figura 2. Productos de PCR amplificados con el ISSR S4: I y | |) Marcador de peso molecular Gene Ruler ( I KB), 2) 60 ng CTABI, 3) 60 ng WIZARD3, 4) 60 ng WIZARDINVITRO2, 5) 60 ng WIZARDINVER2, 6) 60 ng CTABINVER2.

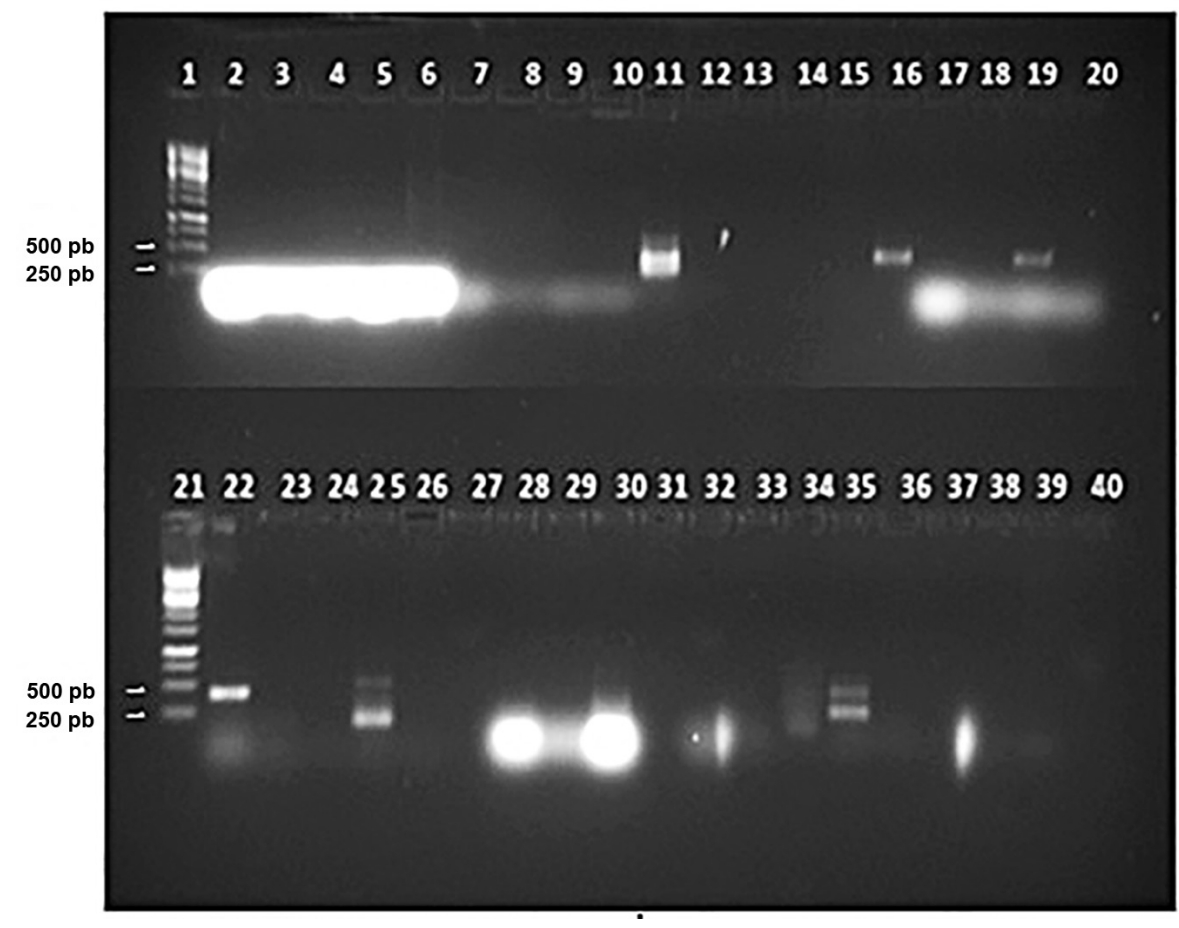

Figura 3. Productos de PCR amplificados con los ISSR S5, SIO, SII, SI2 y SI5: I y 2 I) Marcador de peso molecular Gene Ruler, 2 a 6) Muestras I a $5^{\text {a }}$ Multiplex I, 7- I I) Muestras I a 5 con ISSR S3, 12-16) Muestras I a 5 con ISSR S4, 17-22) Muestras I a 5 con ISSR S5, 23-27) Muestras I a 5 con ISSR SI0, 28-32) Muestras I a 5 con ISSR SII, 33-37) Muestras I a 5 con ISSR SI2, 38-40) Muestras 3 a 5 con ISSR SI 5.

a) Las muestras I a 5 corresponden a CTABI, WIZARD3, WIZARDINVITRO2, WIZARDINVER2 y CTABINVER2, respectivamente. b) Ensayo para el establecimiento de un protocolo de PCR Multiplex, utilizando los imprimadores ISSR S3, S4, S5, SIO, SII, SI2 y SI5. 
Se realizó una nueva electroforesis con los productos de las reacciones $\mathrm{S} 5, \mathrm{SI} 0, \mathrm{SI} I, \mathrm{SI} 2$ y $\mathrm{SI} 5$ para las muestras de ADN 3 y 5 (WIZARDINVITRO2 y CTABINVER2), ya que fueron las únicas que generaron bandas de amplificación visibles, para la mayoría de los ISSR, como se presenta en la figura 4.

Tal y como se aprecia en las figuras anteriores, las muestras WIZARDINVITRO2 (3) y CTABINVER2 (5), al ser las que presentaron bandas amplificadas con el uso de los ISSR, se tomaron como parámetro para la comparación entre los marcadores, para determinar cuáles serían los más adecuadas para la caracterización de materiales de S. rebaudiana. En este caso, los ISSR S5 y SII generaron bandas solo en una de las muestras, por lo que quizá representan una mejor opción para la detección de polimorfismos en esta especie, no obstante, esta afirmación debe constatarse entre un número mayor

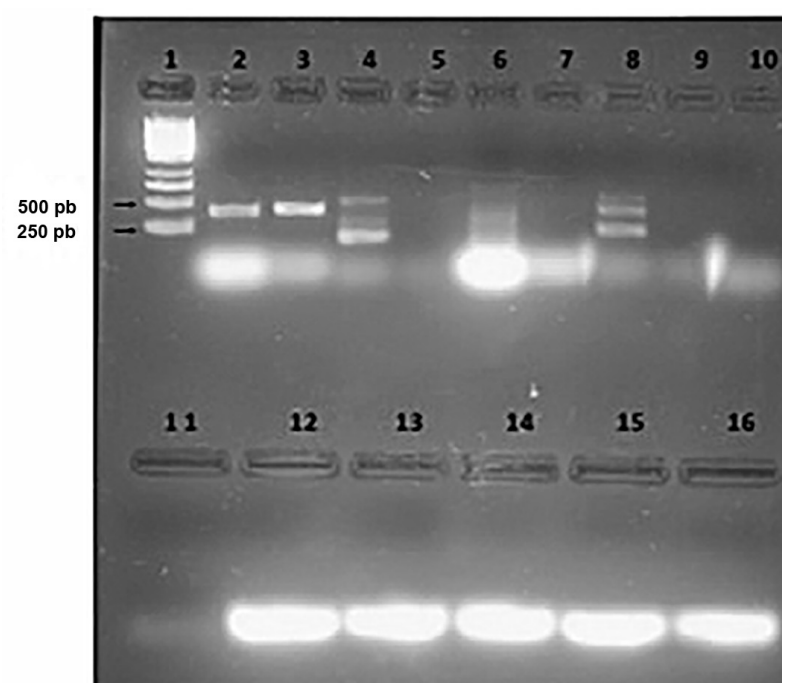

Figura 4. Productos de PCR amplificados con los ISSR S5, SI0, SII, SI2 y SI5: I) Marcador de peso molecular Gene Ruler ( $\mathrm{Kb}$ ), 2 y 3 ) Muestras 3 y $5^{\text {a }}$ con ISSR S5, 4 y 5) Muestras 3 y 5 con ISSR SI0, 6 y 7) Muestras 3 y 5 con ISSR SII, 8 y 9) Muestras 3 y 5 con ISSR SI2, I 0 y II) Muestras 3 y 5 con ISSR SI5, 12-16) Muestras I a 5 Multiplex ${ }^{\text {I. }}$.

a) Las muestras I a 5 corresponden a CTABI, WIZARD3, WIZARDINVITRO2, WIZARDINVER2 y CTABINVER2, respectivamente. b) Ensayo para el establecimiento de un protocolo de PCR Multiplex, utilizando los imprimadores ISSR S3, S4, S5, SIO, SII, SI2 y SI5. de muestras, preferiblemente de diversas variedades de $S$. rebaudiana. Lo anterior también se aplica para la comprobación de que el ISSR SI 5 es capaz de amplificar segmentos de ADN de Stevia, ya que en esta investigación ninguna de las cinco muestras analizadas se amplificó con este imprimador.

Si se considera que los ISSR son marcadores universales, se podría identificar individualmente cada cultivar de Stevia que vaya a destinarse a la producción comercial de esteviósidos, pero sigue siendo necesario determinar los perfiles por cada cebador individualmente, para un determinado número de variedades, para poder definir un índice de genotipo.

\section{Bibliografía}

Aga, E., Bekele, E. \& Bryngelsson,T. (2005). Inter-simple sequence repeat (ISSR) variation in forest coffee trees (Coffea arabica L.) populations from Ethiopia. Genética, 124, 21 3-221.

Azofeifa, A. (2006). Uso de marcadores moleculares en plantas; aplicaciones en frutales del trópico. Agronomía mesoamericana, 17(2), 221-242.

Chávez, M. \& Chavarría, E. (20II). Stevia: endulzante natural de gran potencial comercial. CR: LAICA.

Chia, J. (2009). Caracterización molecular mediante marcadores ISSR de una colección de 50 árboles clonales e híbridos de cacao (Theobroma cacao L.) de la UNAS-Tingo María. Tesis de licenciatura. Universidad Nacional Mayor de San Marcos, Lima.

Collette, B., McDowell, J. \& Graves, J. (2006). Phylogeny of recent billfishes (Xiphioidei). Bulletin of Marine Science, 79(3), 455468

Heikal, H., Badawy O. \& Hafez, A. (2008). Genetic relationships among some Stevia (Stevia rebaudiana Bertoni): Accessions Based on ISSR Analysis. Research Journal of Cell and Molecular Biology, 2(I), I-5.

Hiesh, H., Chai, T. \& Hwang, D. (2005). Rapid PCR-RFLP method for the identification of 5 billfish species. Journal of Food Science, 70(4), 246-249.

Ivanova, N., Dewaard, J. \& Herbert, P. (2006). An inexpensive, automation-friendly protocol for recovering high-quality DNA. Molecular Ecology Notes, 6(4), 998- 1002.

Karaköse, H., Rakesh, J. \& Kuhnert, N. (20I I). Characterization and Quantification of Hydroxycinnamate Derivatives in Stevia rebaudiana Leaves by LC-MSnt. Journal of Agricultural and Food Chemistry, 59, 10143-10150.

Lin, W., Shiau, C. \& Hwang, D. (2005). Identification of four Thunnus tuna species using mitochondrial cytochrome b gene sequence and PCR-RFLP analysis. Journal of Food and Drug Analysis, $13(4), 382-387$.

López, G. (2007). Detección molecular de Helicobacter pylori. Informe de Trabajo Final de Graduación para optar por el 
grado de Bachiller en Ingeniería en Biotecnología. Cartago, CR: Instituto Tecnológico de Costa Rica.

Masumbuko, L. \& Bryngelsson, T. (2006). Inter simple sequence repeat (ISSR) analysis of diploid coffee species and cultivated Coffea arabica L. from Tanzania. Genetic Resources and Crop Evolution, 53, 357-366.

Prakash,V., Upreti, M. \& Prakash, I. (20 I I). Structures of the novel a-glucosyl linked diterpene glycosides from Stevia rebaudiana. Carbohydrate Research, 2034-2038.

Sambrook, J., \& Rusell, D. (200 I). Molecular Cloning: A Laborary Manual. 3 ed. NY: CSHL Press.

Sánchez, N. \& Jiménez, V. (2009). Técnicas moleculares para la detección de variantes somaclonales. Agronomía Mesoamericana, 20(I), |35-15|.
Shreedar, R., Venkatachalam, L., Thimmaraju, N., Bhagyalakhmi, N., Narayan, M. \& Ravishankar, G. (2008). Direct organogenesis from leaf explants of Stevia rebaudiana and cultivation in bioreactor. Biologia Plantarum, 52 (2), 355-360.

Wilfinger, W., Mackey, K. \& Chomcynski, P. (1997). Effect of pH and ionic strength on the spectrophotometric assessment of nucleic acid purity. Biotechniques, 22(3), 474-481.

Yao, Y., Ban, M. \& Brandle, J. (1999). A genetic linkage map for Stevia rebaudiana. Genome, 42, 657-661.

Zietkiewicz, E., Rafalski, A. \& Labuda, D. (1994). Genome fingerprinting by simple sequence repeats (SSR)-anchored polymerase chain reaction amplification. Genomics, 20, 176- I83. 